\title{
EVALUACIÓN ESPIROMÉTRICA EN PACIENTES PREQUIRÚRGICOS, HOSPITAL DE CLÍNICAS
}

\section{SPIROMETRIC EVALUATION IN PRE-SURGICAL PATIENTS, CLINICS HOSPITAL}

\section{Alejandra BELLO ${ }^{1}$, Sara ALVISO ${ }^{1}$, Raúl TORNACO ${ }^{1}$, Susana TORRES ${ }^{1}$, Sergio AQUINO².}

${ }^{1}$ Estudiantes de Medicina, Facultad de Ciencias Médicas, Universidad Nacional de Asunción, San Lorenzo - Paraguay.

${ }^{2}$ Profesor Asistente de Neumología, Facultad de Ciencias Médicas, Universidad Nacional de Asunción, San Lorenzo - Paraguay.

Cómo citar este artículo: Bello A, Alviso S, Tornaco R, Torres S, Aquino S. Evaluación espirométrica en pacientes prequirúrgicos, Hospital de Clínicas. Medicina Clínica y Social. 2017;1(1):31-34.

\section{RESUMEN}

Introducción: La espirometría es una prueba fundamental en la evaluación funcional respiratoria. Esta prueba es utilizada frecuentemente en la práctica clínica y en estudios de poblaciones, así como un importante predictor de complicaciones respiratorias. Objetivo: determinar la frecuencia de patrones patológicos en espirometrías prequirúrgicas. Metodología: Fue un estudio descriptivo, transversal, no probabilístico de casos consecutivos. La población accesible la constituyeron los pacientes que consultaron en la Cátedra de Neumología del Hospital de Clínicas para evaluación espirométrica prequirúrgica. Se excluyó a aquellos pacientes con datos incompletos. Para esta investigación se incluyó un registro de 970 fichas de pacientes. Resultados: Se observó que el 74,5\% presentó una espirometría dentro de los límites normales, 10,9\% presentó patrón restrictivo y 14,5\% presentó algún grado de obstrucción. En cuanto al riesgo prequirúgico se encontró que el $86 \%$ presenta bajo riesgo y el $14 \%$ un riesgo medio. Discusión: la mayoría de los pacientes que se realizaron espirometrías en el contexto de una evaluación prequirúrgica tuvieron resultados dentro de los límites normales, así como bajo riesgo quirúrgico según la escala utilizada. Lo anterior está en concordancia con un estudio realizado en México, donde el $67 \%$ de los pacientes sometidos a espirometría antes de una cirugía presentaron un patrón normal.

Palabras clave: Espirometría; Cirugía; Evaluación prequirúrgica; Neumología.

\section{ABSTRACT}

Introduction: Spirometry is a fundamental test in functional respiratory evaluation. This test is frequently used in clinical practice and in population studies, as well as an important predictor of respiratory complications. Objective: to determine the frequency of pathological patterns in preoperative spirometries. Methodology: It was a descriptive, transversal, non-probabilistic study of consecutive cases. The accessible population was the patients who consulted in the Pneumonology Department at Clinics Hospital for preoperative spirometric evaluation. Patients with incomplete data were excluded. For this investigation, a record of 970 patients was included. Results: It was observed that $74.5 \%$ presented spirometry within the normal limits, $10.9 \%$ presented restrictive pattern and $14.5 \%$ presented some degree of obstruction. Regarding the pre-surgical risk, $86 \%$ had low risk and $14 \%$ had an average risk. Discussion: The majority of patients who underwent spirometry in the context of a preoperative evaluation had results within normal limits, as well as low surgical risk according to the scale used. This is in agreement with a study carried out in Mexico, where $67 \%$ of patients undergoing spirometry before surgery had a normal pattern.

Keywords: Spirometry; Surgery; Pre-Surgical Evaluation; Pneumonology. 


\section{INTRODUCCIÓN}

La espirometría es una prueba fundamental en la evaluación funcional respiratoria. Esta prueba es utilizada frecuentemente en la práctica clínica y en estudios de poblaciones. Entre los diversos índices derivados de una espiración forzada, el volumen espiratorio forzado en el primer segundo de la maniobra $\left(\mathrm{VEF}_{1}\right)$ y la capacidad vital forzada (CVF) son los más usados debido a su buena reproducibilidad, facilidad de medición, y su grado de correlación con la etapa de la enfermedad, condición funcional, morbilidad y mortalidad (1).

La espirometría mide la CVF, el $\mathrm{VEF}_{1}$ y la relación $\mathrm{VEF}_{1} / \mathrm{CVF}$, que es la proporción de la CVF exhalada en el primer segundo de esta maniobra (2). Es considerada una prueba de máximo esfuerzo, es decir, requiere la completa comprensión neurológica y cooperación por el sujeto para realizarla en condiciones adecuadas y repetibles, con tecnología adecuada (3).

De acuerdo a las guías de interpretación de espirometría de la American Thoracic Society/European Respiratory Society, en la respuesta broncodilatadora incrementos de $12 \%$ y $200 \mathrm{ml}$ en el VEF 1 y CVF comparados con el valor basal son sugestivos de una respuesta broncodilatadora significativa (4). La espirometría tiene un papel indiscutible en la clínica como un estudio de valoración de la integridad de la mecánica pulmonar, y dentro de la valoración preoperatoria. Si bien la espirometría como prueba de valoración de la función pulmonar no proporciona límites exactos a partir de los cuales un paciente que va a ser intervenido quirúrgicamente puede ser considerado como inoperable, nos proporciona datos para dividirlos en grupos de alto y bajo riesgo quirúrgico (5).

La identificación de los factores de riesgo y su corrección o mejora, la mejoría en el tratamiento de las complicaciones postquirúrgicas y los avances anestésicos y técnicos, nos han permitido ampliar la oferta quirúrgica a pacientes considerados hasta hace poco tiempo inoperables. Por ello, la evaluación funcional preoperatoria está en continua evolución (6).

Sistemas de puntuación de evaluación de riesgo para la evaluación preoperatoria se utilizan con el fin de estimar la probabilidad de que los pacientes desarrollen complicaciones postoperatorias. El sistema de puntuación ideado por Torrington y Henderson, es capaz de estimar las complicaciones pulmonares y la mortalidad, a través de la clasificación de los pacientes como de bajo, moderado o alto riesgo (7).

\section{METODOLOGÍA}

Fue un estudio descriptivo de corte transversal, no probabilístico de casos consecutivos, llevado a cabo en el Hospital de Clínicas, dependiente de la Universidad Nacional de Asunción durante el 7 de enero del 2015 al 23 de diciembre del 2015. La población enfocada estuvo constituida por todos los pacientes prequirúrgicos; mientras que la población accesible la constituyeron los pacientes que consultaron en la Cátedra de Neumología del Hospital de Clínicas para evaluación espirométrica prequirúrgica en el periodo señalado. Se excluyó a aquellos pacientes con datos incompletos.

El sistema de puntuación de Torrington y Henderson, fue utilizado para estimar las complicaciones pulmonares y la mortalidad, a través de la clasificación de los pacientes como de bajo, moderado o alto riesgo. 
Para esta investigación se incluyó un registro de 970 fichas de pacientes que se realizaron consulta prequirúrgica. Los datos obtenidos fueron archivados utilizando tablas de Microsoft Excel $^{\circledR}$ y posteriormente analizados con el software estadístico Epilnfo versión 7.2 (CDC, Atlanta). Se garantizó el anonimato de los pacientes al momento de la toma de muestras, así como durante el análisis y la presentación de los resultados. En todo momento se respetaron los principios de bioética aplicados a la investigación biomédica.

\section{RESULTADOS}

Se analizó un total de 970 informes de espirometría, de los cuales 340 correspondieron a evaluaciones prequirúrgicas. Se observó que el 74,5\% presentó una espirometría dentro de los límites normales, 10,9\% presentó patrón restrictivo y 14,5\% presentó algún grado de obstrucción.

Teniendo en cuenta las evaluaciones prequirúrgicas se constató que el $40 \%$ correspondió a cirugías de abdomen superior, el $29 \%$ abdomen inferior, $15 \%$ cabeza y cuello, $10 \%$ tórax y $6 \%$ miembros y vascular.

De los pacientes que presentaron un patrón obstructivo; $25 \%$ fueron obstrucción leve, 37,5\% moderado y $37,5 \%$ grave. En cuanto al riesgo prequirúgico se encontró que el $86 \%$ presenta bajo riesgo y el $14 \%$ un riesgo medio.

\section{DISCUSIÓN}

Se observa, según los datos obtenidos, que la mayoría de los pacientes que se realizaron espirometrías en el contexto de una evaluación prequirúrgica tuvieron resultados dentro de los límites normales, así como bajo riesgo quirúrgico según la escala utilizada en el Servicio de Neumología. Lo anterior está en concordancia con un estudio realizado en México, donde el $67 \%$ de los pacientes sometidos a espirometría antes de una cirugía presentaron un patrón normal (8). De acuerdo a los hallazgos la espirometría es solicitada sobre todo en cirugías abdominales y fue en este grupo donde pudimos encontrar la mayor cantidad de patrones patológicos, sin embargo, hay que tener en cuenta que esto pudo deberse a que fue el mayor grupo de cirugía que formó parte de la muestra.

Saber el tipo de cirugía que van a realizarse es parte de la evaluación prequirúrgica, ya que según consensos las cirugías que involucran un mayor riesgo son la torácica, abdominal superior, aórtica, vascular periférica, neurocirugía; por lo que una correcta evaluación del aparato respiratorio es fundamental; este mismo consenso establece que son predictores de complicaciones respiratorias: la edad, la enfermedad obstructiva crónica, tabaquismo reciente, mal estado general, radiografía anormal de tórax (9).

En la investigación existen ciertas limitaciones, como el tipo de estudio y muestreo realizado, ya que no pueden establecerse relaciones entre las variables estudiadas y tampoco pueden generalizarse los resultados obtenidos, por lo que recomendamos estudios posteriores que busquen relacionar variables. Así también, es importante incluir variables sociodemográficas, enfermedad de base, resultado de la cirugía y evaluación posterior a la misma. En nuestra revisión de fichas se notó ausencia de datos sobre la técnica espirométrica y la utilización de broncodilatadores, así mismo, se encontró fichas con indicación de evaluación prequirúrgica, 
sin embargo, no tenían hecha una espirometría. Por lo que recomendamos se realice un llamado de atención para el correcto y completo llenado de las fichas clínicas y que en todas las evaluaciones solicitadas al Servicio se incluya a la espirometría como un método fundamental para el pronóstico del éxito terapéutico y así evitar complicaciones respiratorias posteriores o durante las cirugías.

\section{CONFLICTOS DE INTERÉS Y FUENTE DE FINANCIACIÓN}

Los autores declaran no poseer conflictos de interés. Fuente de financiación: ninguna.

\section{REFERENCIAS BIBLIOGRÁFICAS}

1. Gutiérrez C, Beroíza T, Borzone G, Caviedes I, Céspedes J, Gutiérrez M, et al. Espirometría: Manual de procedimientos. Sociedad Chilena de Enfermedades Respiratorias, 2006. Rev Chil Enferm Respir. 2016;23(1):31-42. http://dx.doi.org/10.4067/S0717$\underline{73482007000100005}$

2. Miller MR, Hankinson J, Brusasco V, Burgos F, Casaburi R, Coates A, et al. Standardization of spirometry. Eur Respir J. 2005;26(2):319-338. http://www.dx.doi.org/10.1183/09031936.05.00034805

3. Hyatt RE, Scanlon PD, Nakamura M. Interpretative strategies for lung function tests. A practical guide. 3a ed. Rochester, MN: Lippincott Williams \& Wilkins;2009.

4. Centers for Disease Control and Prevention [Internet]. Spirometry. [citado el 12 de enero de 2017]. Disponible en: http://www.cdc.gov/nchs/data/nhanes/nhanes 07 08/spirometry.pdf

5. Santos C, Eugenia M, Pacheco A, Elena M, Bermeo M, Gustavo E. Evaluación funcional respiratoria en el pre y post-operatorio. Estudio espirográfico en pacientes del departamento de cirugía del H.V.C.M [Tesis de grado]. Cuenca: Facultad de Ciencias Médicas, Universidad de Cuenca; 1998. [citado el 9 de enero de 2017]. URL.

6. Vaquero-Barrios JM, Escribano-Dueñas AM, Redel-Montero J, Cobos-Ceballos MJ. Evaluación preoperatoria en respiratorio [Internet]. [citado el 9 de enero de 2017]. Disponible en: http://www.neumosur.net/files/EB04-15\%20preoperatorio.pdf

7. Stanzani F, Oliveira MA, Forte V, Faresin SM. Escalas de risco de Torrington e Henderson e de Epstein: aplicabilidade e efetividade nas ressecções pulmonares. J Bras Pneumol. 2005;31(4):292-299. http://dx.doi.org/10.1590/S1806-37132005000400005

8. Mares-Gutiérrez MY, Sánchez-Velázquez LD. Espirometría en la valoración preoperatoria en el Hospital General de México. Revista Médica del Hospital General de México. 2013;76(1):23-28. URL.

9. Falconi M, Barisani JL, Levín R, Sampó E, Vidal L, Amuchástegui LM, et al. Consenso de evaluación del riesgo cardiovascular en cirugía no cardíaca. Rev Argent Cardiol. 2005;73(5):381-396. URL. 